\title{
Parity Versions of 2-Connectedness
}

\author{
C. Little \\ Institute of Fundamental Sciences \\ Massey University \\ Palmerston North, New Zealand \\ c.little@massey.ac.nz
}

\author{
A. Vince \\ Department of Mathematics \\ University of Florida \\ Gainesville, FL., USA \\ vince@math.ufl.edu
}

Submitted: Nov 16, 2005; Accepted: Oct 18, 2006; Published: Oct 31, 2006

Mathematics Subject Classification: 05C40, 05C38

\begin{abstract}
This paper introduces parity versions of familiar graph theoretic results, in particular results related to 2-connectedness. The even and odd circuit connected graphs are characterized. The realizable, even-realizable, alternating-realizable, dual realizable and dual even-realizable graphs are classified.
\end{abstract}

\section{Introduction}

Connectivity is a basic concept in graph theory. In particular, 2-connectedness plays an indispensable role throughout the subject. The intent of this paper is to provide parity versions of a few results concerning 2-connectedness. To simplify statements it will be assumed throughout that all graphs are connected. The definition of 2-connectivity for such a graph is as follows. A connected graph $G$ with at least 3 vertices is 2-connected if the removal of at least 2 vertices is required to disconnect $G$. Concerning notation, the vertex and edge sets of a graph $G$ are denoted $V(G)$ and $E(G)$, respectively. A circuit in $G$ is the edge set of a non-empty connected subgraph in which each vertex is of degree 2 . 
A circuit or path will be considered to be a set of edges. A circuit or path is even or odd according to its cardinality. For any set $E$ of edges of $G$, the subgraph induced by $E$ is denoted $G[E]$.

A classic result is that a graph is 2-connected if and only if, for every pair of edges, there is a circuit containing them. A related concept is that of circuit connectedness. Call a graph $G$ circuit connected if, for any partition of the edges of $G$ into two non-empty parts $S$ and $T$, there is a circuit $C$ such that

$$
C \cap S \neq \emptyset \quad \text { and } \quad C \cap T \neq \emptyset .
$$

It is easy to show that a graph $G$ is 2-connected if and only if it is circuit connected. Call a graph $G$ even (odd) circuit connected if, in the above definition, $C$ must have even (odd) length. The graphs that are even (odd) circuit connected are characterized in Section 2. The notion of (even) circuit connectedness originated in [1] where it played an integral part in the proof of the main theorem. Note that the even (odd) circuit connectedness property and the property that every pair of edges is contained in an even (odd) circuit are not equivalent. Figure 1 shows a graph that is both even and odd circuit connected, but the two "thick" edges are not, in the first case, contained in any odd circuit and, in the second case, in any even circuit.
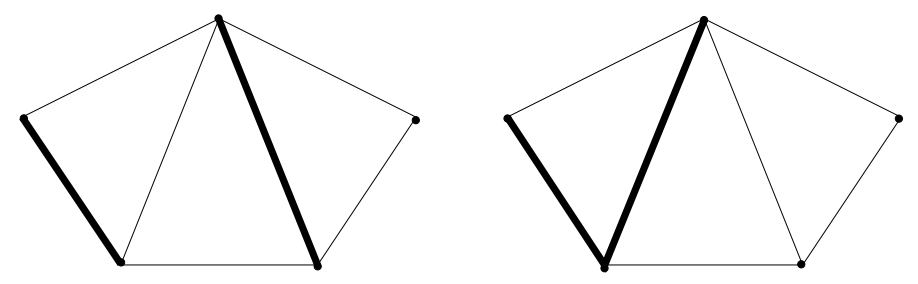

Figure 1: Even and odd circuit connected.

Another context in which parity in a 2-connected graph plays a role is that of realizable graphs $[1,3]$. To each circuit of a 2-connected graph $G$ assign a parity - even or odd. If $\mathcal{C}$ denotes the set of all circuits, then this assignment can be considered a function

$$
f: \mathcal{C} \rightarrow \mathbb{Z}_{2}
$$

where 0 is even and 1 is odd. Call $f$ realizable if there exists a set $E$ of edges of $G$ such that

$$
|C \cap E| \equiv f(C) \quad(\bmod 2)
$$

for every circuit $C \in \mathcal{C}$. Call $G$ realizable if every function $f: \mathcal{C} \rightarrow \mathbb{Z}_{2}$ is realizable. The set $\mathcal{C}$ generates the cycle space of $G$. Likewise the set $\mathcal{C}_{0}$ of even length circuits generates the even cycle space. Call $G$ even-realizable if every function $f: \mathcal{C}_{0} \rightarrow \mathbb{Z}_{2}$ is realizable. The even-realizable graphs are characterized in [1] in terms of forbidden subgraphs. Section 3 of this paper provides a complete classification of the even-realizable graphs with a short 
proof that is independent of previous results. Also contained in Section 3 is an analogous classification with respect to the cocycle space, a classification of the graphs that are the subject of [4]. The proof is based on a dual version of the well-known Ear Decomposition Theorem for 2-connected graphs.

The matter of realizability arises in connection with the study of Pfaffian graphs $[2,4,5,6]$. A circuit in a graph $G$ that admits a 1-factor is alternating if it is a symmetric difference of two 1-factors. In other words, a circuit $C$ in $G$ is alternating if $G-V(C)$ has a 1-factor. Alternating circuits are clearly of even length. An alternating circuit in a directed graph is evenly or oddly oriented according to the parity of the number of its edges that are oriented in agreement with a preassigned sense. The orientation of $G$ is said to be Pfaffian if every alternating circuit is oddly oriented. A graph is Pfaffian if it admits such an orientation. The Pfaffian property of graphs is related to various properties of $0-1$ matrices and of hypergraphs [7]. Pfaffian bipartite graphs have been characterized in [6] and Pfaffian near bipartite graphs in [2, 5], but no characterization of Pfaffian graphs is known as yet.

In order to try to find a Pfaffian orientation for a given graph $G$, one might begin by trying an arbitrary orientation. If this orientation is not Pfaffian, then some alternating circuits are evenly oriented. If $\mathcal{A}$ is the set of all alternating circuits, one may define a function $f: \mathcal{A} \rightarrow \mathbb{Z}_{2}$ such that $f(A)$ has opposite parity to the orientation of $A$ for each alternating circuit $A$. In order to obtain a Pfaffian orientation, one must then choose a set $E$ of edges such that $|E \cap A| \equiv f(A)(\bmod 2)$ for each $A \in \mathcal{A}$. Reorientation of the edges in $E$ will then secure the required Pfaffian orientation. If $E$ exists, then we say that $f$ is alternating-realizable. The graph $G$ is alternating-realizable if every function $f: \mathcal{A} \rightarrow \mathbb{Z}_{2}$ is alternating-realizable. This paper includes a characterization of alternating-realizable graphs.

\section{Circuit connectedness}

The graphs that are even (odd) circuit connected are characterized in this section. As noted in the introduction, this is not equivalent to characterizing the graphs for which every pair of edges are contained in an even (odd) circuit. A characterization in the latter case follows routinely from results in [8] for 3-connected graphs.

An ear of a graph $G$ is a maximal path whose internal vertices have degree 2 in $G$. Let $P$ be an ear of $G$ and let $H$ be the subgraph of $G$ obtained by deleting the internal vertices of $P$. Then $G$ is said to be obtained from $H$ by an ear addition. An ear decomposition of $G$ is a sequence $G_{0}, G_{1}, \ldots, G_{n}$ of subgraphs of $G$ such that $G_{0}=K_{2}, G_{n}=G$ and $G_{i}$ is

obtained from $G_{i-1}$ by an ear addition for each $i>0$. In the proof of Theorem 3 in this section the following well known facts will be used.

1. A graph $G$ with at least 3 vertices is 2 -connected if and only if $G$ has an ear decomposition. 
2. Any graph obtained from a 2-connected graph by an ear addition is also 2-connected.

3. Let $H$ be a proper subgraph of a 2-connected graph $G$ with $E(H) \neq \emptyset$. If $e \in$ $E(G)-E(H)$, then $e$ is contained in a subgraph $K$ of $G$ obtained from $H$ by a single ear addition.

The following lemmas will also be used.

Lemma 1 Let $C$ be an odd circuit in a 2-connected graph $G$ and let $e \in E(G)-C$. Then $G$ has an even (odd) circuit through e containing an edge of $C$.

Proof: Choose $f \in C$. By 2-connectedness $G$ has a circuit $D$ through $e$ and $f$. Some subset of $D$ must be an ear $P$ in $G[C \cup D]$ containing $e$. Then $P \cup C$ includes two circuits through $e$. Since $C$ is odd, one of these circuits is even and the other is odd. Both contain an edge of $C$.

Lemma 2 Let $H$ and $K$ be even (odd) circuit connected subgraphs of a 2-connected graph $G$ and suppose $E(H) \cap E(K) \neq \emptyset$. Then $G[E(H) \cup E(K)]$ is even (odd) circuit connected.

Proof: Let $\{S, T\}$ be a partition of $E(H) \cup E(K)$ and without loss of generality suppose that $S \cap E(H) \neq \emptyset$. If also $T \cap E(H) \neq \emptyset$ then some even (odd) circuit meets both $S$ and $T$ by the even (odd) circuit connectedness of $H$. Therefore we may assume that $\emptyset \neq T \subseteq E(K)-E(H)$. But then $S \cap E(K) \neq \emptyset$, since $E(H) \cap E(K) \neq \emptyset$, and so there is an even (odd) circuit meeting $S$ and $T$ by the even (odd) circuit connectedness of $K$.

Call a graph $G$ twin bipartite if $G$ is the union of two bipartite subgraphs $H$ and $H^{\prime}$ such that

1. $E(H) \cap E\left(H^{\prime}\right)=\emptyset$ and,

2. $V(H) \cap V\left(H^{\prime}\right)=\{u, v\}$, where $u$ and $v$ lie in the same partite set of $H$ and in different partite sets of $H^{\prime}$.

Theorem 3 A 2-connected graph $G$ is

1. odd circuit connected if and only if $G$ is not bipartite,

2. even circuit connected if and only if $G$ is not twin bipartite.

Proof: The proof in one direction is easy in both cases. If $G$ is bipartite, then $G$ has no odd circuits, hence cannot be odd circuit connected. If $G$ is twin bipartite, any circuit that contains edges of both $E(H)$ and $E\left(H^{\prime}\right)$ must be odd. Therefore taking the bipartition $\{S, T\}$ where $S=E(H)$ and $T=E\left(H^{\prime}\right)$ shows that $G$ is not even circuit connected.

Consider statement (2) in the other direction. Assume that $G$ is 2-connected but not even circuit connected. We must show that $G$ is twin bipartite. Not being even 
circuit connected, $G$ has an odd circuit $C$. If $G=G[C]$ then $G$ is twin bipartite. (Take $H=G[\{e\}]$ for some edge $e$ of $C$.)

If, on the other hand, there is an edge $e \in E(G)-C$, then Lemma 1 implies the existence of an even circuit and hence the existence of a non-empty even circuit connected subgraph. Let $H$ be a maximal even circuit connected subgraph. Recall that $H$ is 2connected because it is circuit connected. But $H$ must be a proper subgraph of $G$ as $G$ is not even circuit connected.

Let $H^{\prime}=G[E(G)-E(H)]$. There is no even circuit $Q$ in $G$ meeting both $E(H)$ and $E\left(H^{\prime}\right)$. Otherwise $G[Q]$ would be even circuit connected, and by Lemma 2 so would $G[E(H) \cup Q]$, contradicting the maximality of $H$. Both $H$ and $H^{\prime}$ are bipartite. Otherwise we can choose an odd circuit from one and an edge from the other and apply Lemma 1 to obtain an even circuit in $G$ meeting $E(H)$ and $E\left(H^{\prime}\right)$.

Clearly $E(H) \cap E\left(H^{\prime}\right)=\emptyset$. It now suffices to verify condition (2) for $H$ and $H^{\prime}$ in the definition of twin bipartite. Since $H$ is a proper subgraph of the 2-connected graph $G$, there is a path $P$ in $H^{\prime}$ having exactly two vertices $u, v$ in common with $H$. There is also a path $P^{\prime}$ in $H$ joining $u$ and $v$. Consequently there exists a circuit $A=P \cup P^{\prime}$ in $E(H) \cup P$ meeting both $E(H)$ and $E\left(H^{\prime}\right)$. By what was proved above, the circuit $A$ must be odd.

We next claim that $V(H) \cap V\left(H^{\prime}\right)=\{u, v\}$. If not, there is a $w \neq u, v$ such that $w \in V(H) \cap V\left(H^{\prime}\right)$. Let $e$ be an edge of $H^{\prime}$ incident with $w$. There must exist a 2connected subgraph $K$ of $G$ that contains $e$ and is obtained from the 2-connected graph $G[E(H) \cup P]$ by an ear addition. Note that $K$ contains the odd circuit $A$ not containing $e$. By Lemma 1 there exists an even circuit $C$ in $K$ containing $e$. But by the definition of $e$, the even circuit $C$ must meet both $E(H)$ and $E\left(H^{\prime}\right)$, a contradiction to what was proved above.

To complete the proof that $G$ is twin bipartite, it suffices to show that $u$ and $v$ lie in the same partite set of one of $H$ or $H^{\prime}$ and in different partite sets of the other. Since $A$ is an odd circuit containing $u$ and $v$ this must be the case.

Finally consider part (1) of the theorem. By way of contradiction, suppose $G$ is not odd circuit connected and not bipartite. Then $G$ has an odd circuit $C$, and $G[C]$ is a non-empty odd circuit connected subgraph of $G$. Choose $H$ to be a maximal odd circuit connected subgraph of $G$. Since $H$ is circuit connected, it is 2-connected. As $G$ is not odd circuit connected, $H \neq G$. By Lemma 1 there is an odd circuit $A$ such that $A \cap E(H) \neq \emptyset$ and $A \cap(E(G)-E(H)) \neq \emptyset$. But by Lemma $2, G^{\prime}=G[E(H) \cup A]$ is odd circuit connected, contradicting the maximality of $H$.

\section{$3 \quad$ Realizable graphs}

A graph is allowed to have vertices of degree 2 and multiple edges. A subdivision of a graph $G$ is obtained from $G$ by adding any number of vertices of degree 2 to existing 
edges. An even subdivision of $G$ is obtained from $G$ by adding an even number of vertices of degree 2 to each of any number of existing edges. Two graphs are said to be (even) equivalent if they have a common (even) subdivision. A graph $H$ is said to be (even) contained in graph $G$ if $H$ is (even) equivalent to a subgraph of $G$.

Lemma 4 A graph is (even) realizable if and only if there exists no linearly dependent set of circuits in its (even) cycle space. A graph is alternating-realizable if and only there exists no linearly dependent set of alternating circuits.

Proof: Let $E$ denote the set of edges and $\mathcal{C}$ the set of (even) circuits of $G$. Let $M$ be a $(0,1)$ matrix whose rows are indexed by $\mathcal{C}$ and columns by $E$ and whose entry $m_{C, e}=1$ if and only if $e \in C$. By definition $G$ is (even) realizable if and only if $\operatorname{dim}$ (column space $M$ ) $=$ $|\mathcal{C}|$. But $\operatorname{dim}($ column space $M)=\operatorname{dim}($ row space $M)$, and $\operatorname{dim}($ row space $M)=|\mathcal{C}|$ if and only if there are no dependencies among the rows. No dependencies among the rows means no linearly dependent sets of circuits in its (even) cycle space. The same proof holds for the alternating-realizable case.

The following two results follow immediately from Lemma 4.

Corollary 5 If $G$ is (even) realizable and $H$ is (even)-contained in $G$, then $H$ is (even) realizable.

In Figure $2 \mathrm{~F}$, the edges $\{a, b\},\{c, d\},\{e, f\}$ are meant to represent paths of any length, including length 0 . It is possible, for example, that $a=b$.

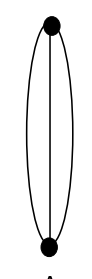

A

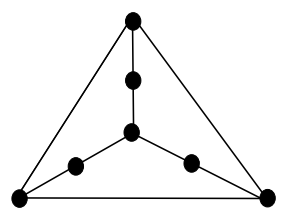

D

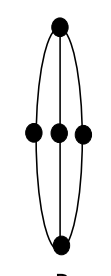

B

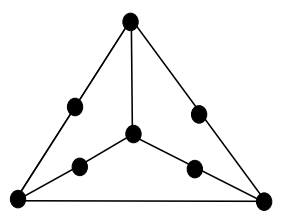

E

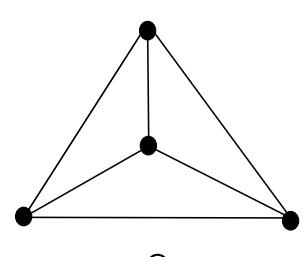

C

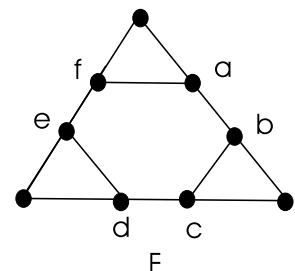

Figure 2: Forbidden graphs.

Corollary 6 None of the graphs in Figure 2 is even-realizable. 
Call each of the graphs in Figure 2 a forbidden graph.

Corollary 7 If $G$ even-contains a forbidden graph, then $G$ is not even-realizable.

Define three families of graphs $\mathcal{G}_{0}, \mathcal{G}_{1}, \mathcal{G}_{2}$ as follows. The family $\mathcal{G}_{0}$ consists of all circuits (even and odd). The family $\mathcal{G}_{1}$ consists of all graphs obtained from an odd circuit by replacing any number of single edges by copies of the graph in Figure 3A, and any number of pairs of consecutive edges by copies of the graph in Figure 3B. An examples of a graph in $\mathcal{G}_{1}$ also appears in Figure 3. The family $\mathcal{G}_{2}$ is represented by the graph in Figure 4 , where the line joining vertices $u$ and $v$ represents a path of any length, even or odd, including the empty path, in which case $u=v$. The same is true for the line joining $u^{\prime}$ and $v^{\prime}$. A graph even-equivalent to a graph from $\mathcal{G}_{0}, \mathcal{G}_{1}$ or $\mathcal{G}_{2}$ will be referred to as a graph of type $\mathcal{G}_{0}, \mathcal{G}_{1}$ or $\mathcal{G}_{2}$, respectively.

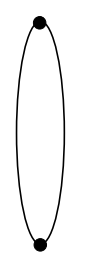

A

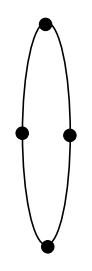

B
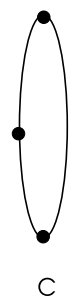

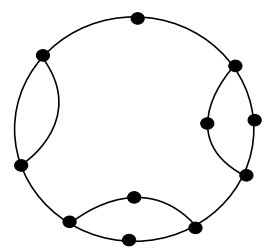

Figure 3: An example of a graph in $\mathcal{G}_{1}$.

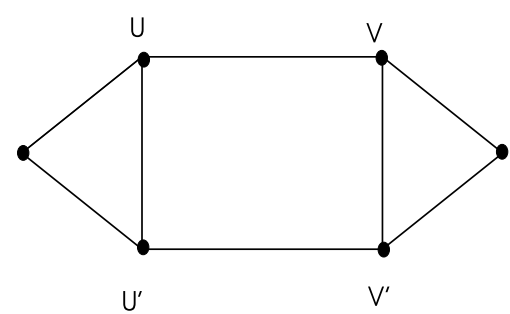

Figure 4: The graphs $\mathcal{G}_{2}$.

Concerning alternating-realizability only edges that belong to a 1-factor are relevant. For this reason the notion of 1-extendability is required. A graph is said to be 1-extendable if for each edge $e$ there is a 1 -factor that contains $e$.

Theorem 8 Let $G$ be a 2-connected graph.

1. $G$ is realizable if and only if $G$ is a circuit.

2. $G$ is even-realizable if and only if $G$ is of type $\mathcal{G}_{0}, \mathcal{G}_{1}$ or $\mathcal{G}_{2}$. 
3. A 1-extendable graph $G$ is alternating-realizable if and only if $E(G)$ is an even circuit or $G$ is isomorphic to $K_{2}$.

Proof: Concerning statement (1), if $G$ is a single circuit, then $G$ is obviously realizable. In the other direction, if $G$ is 2-connected but not a circuit, then $G$ contains the graph $2 \mathrm{~A}$. But $2 \mathrm{~A}$ is not realizable. By Corollary $5, G$ is also not realizable.

Concerning statement (2), if $G$ is a graph of type $\mathcal{G}_{0}, \mathcal{G}_{1}$ or $\mathcal{G}_{2}$, then it follows from Lemma 4 that $G$ is even-realizable. In the other direction, assume that $G$ is evenrealizable. We must show that $G$ is of type $\mathcal{G}_{0}, \mathcal{G}_{1}$ or $\mathcal{G}_{2}$. The first step is to show that certain configurations cannot appear in $G$.

1. $G$ contains no $K_{4}$.

Suppose, by way of contradiction, that $G$ does contain a subdivision $J$ of $K_{4}$. Any embedding of $J$ in the plane has four faces. If one of the face boundaries has even length, then at least two of them have even length since the sum of the lengths of the face boundaries is even. But any two face boundaries share an edge. Therefore in this case $G$ even-contains 2A or 2B and hence, by Corollary 7, $G$ cannot evencontain $J$. We may therefore suppose that all the face boundaries of $J$ have odd length. One possibility is an even subdivision of the graph $2 \mathrm{C}$. The only other possibilities are obtained from an even subdivision of the graph $2 \mathrm{C}$ by choosing an even number of edges from each face boundary of $K_{4}$ and changing the parity of the lengths of the paths of $J$ corresponding to the chosen edges. The set of chosen edges of $K_{4}$ is a non-empty cocycle of $K_{4}$ since the face boundaries span the cycle space of $K_{4}$. The resulting graphs are therefore even subdivisions of those of 2D and $2 \mathrm{E}$, again forbidden by Corollary 7 .

Let $\left\{v_{i}, v_{j}\right\}$ and $\left\{v_{s}, v_{t}\right\}$ be two chords, with no vertex in common, of a circuit with successive vertices $v_{0}, v_{1}, \ldots, v_{n}=v_{0}$. Without loss of generality assume that $0=i<s<$ $j$. These chords are said to be crossing if $j<t$. Three distinct chords $\left\{v_{i}, v_{j}\right\},\left\{v_{p}, v_{q}\right\}$ and $\left\{v_{s}, v_{t}\right\}$ of a circuit, no pair crossing, are called parallel if the indices cannot be assigned so that $i<j \leq p<q \leq s<t$.

2. $G$ contains no circuit with crossing chords.

Otherwise $G$ would contain a $K_{4}$ which is impossible by statement (1).

3. $G$ contains no circuit with 3 parallel chords.

Indeed, let $J$ be a subdivision of a graph induced by a circuit and three parallel chords. The graph is drawn in Figure 5 where the labels on the edges indicate the lengths of the corresponding paths in $J$. In the following argument, equality denotes congruence modulo 2. If $a \neq b$ and $d \neq e$, then we may choose $x \in\{a, b\}$ and $y \in\{d, e\}$ such that $f+x+h=g+y+i=c$, so that $G$ even-contains 2A or 2B. We may therefore assume without loss of generality that $a=b$. Then $d=e$, 
for otherwise we may choose $y \in\{d, e\}$ so that $a=b=f+g+y+i+h$ and hence $G$ even-contains 2A or 2B. Similarly $g+d+i=c$; otherwise we may choose $y \in\{c, g+d+i\}$ so that $a=b=f+y+h$. By symmetry $f+b+h=c$, and yet again we find that $G$ even-contains $2 \mathrm{~A}$ or $2 \mathrm{~B}$. Thus in every case we obtain a contradiction to Corollary 7.

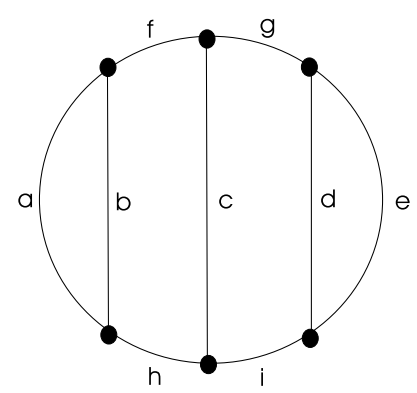

Figure 5: A circuit with 3 parallel chords.

4. $G$ can contain no circuit with one edge replaced by $3 \mathrm{~A}$ or $3 \mathrm{~B}$ and another edge replaced by $3 \mathrm{C}$.

Otherwise $G$ would even-contain a forbidden graph $2 \mathrm{~A}$ or $2 \mathrm{~B}$.

5. G can contain no circuit with three edges replaced by 3C.

Otherwise $G$ would even-contain the forbidden graph $2 \mathrm{~F}$.

Since $G$ is 2-connected it has an ear decomposition. It will now be shown, by induction on the number of ears in an ear decomposition of $G$, that $G$ must be of type $\mathcal{G}_{0}, \mathcal{G}_{1}$ or $\mathcal{G}_{2}$. If there is just one ear in the ear decomposition, then $G$ is in $\mathcal{G}_{0}$. Let $G^{\prime}$ be the second to last stage in the ear decomposition. The addition of one more ear to $G^{\prime}$ will give $G$. Since $G^{\prime}$ is a subgraph of $G$, it is even-realizable by Corollary 5. By the induction hypothesis $G^{\prime}$ is of type $\mathcal{G}_{0}, \mathcal{G}_{1}$ or $\mathcal{G}_{2}$.

Suppose $G^{\prime}$ is of type $\mathcal{G}_{0}$. Addition of an ear results in a graph with two vertices of degree 3 joined by three independent paths. If the lengths of these paths are of equal parity, then $G$ even-contains $2 \mathrm{~A}$ or 2B. Otherwise $G$ is of type $\mathcal{G}_{1}$.

In the remaining cases $G^{\prime}$ has a circuit $C$ of odd length containing two vertices joined by three independent paths $P, Q, R$, where $P \cup Q=C$. Any ear added to the graph $G^{\prime}[C \cup R]$ must join two vertices of the same such path, since $G$ contains no $K_{4}$.

Suppose first that there exists a path $S \neq R$ joining two vertices of $C$ and having no internal vertex in $C$. Then $S$ must be parallel to $R$. The graph $G^{\prime}[C \cup R \cup S]$ is shown in Figure 6, where $W, X, Y, Z$ denote disjoint paths in $C$ whose union is $C$. An ear added to this graph cannot join a vertex of $R$ to a vertex of $S$ because $G$ contains no $K_{4}$. Nor can it join a vertex of $X$ to a vertex of $Z$ since no circuit of $G$ has three parallel chords. 
Similarly it cannot join two vertices of $Y$. By symmetry and the result of the previous paragraph, we may therefore assume that it joins two vertices of $X$. It then follows from (4) and (5) that $G$ is of type $\mathcal{G}_{1}$.

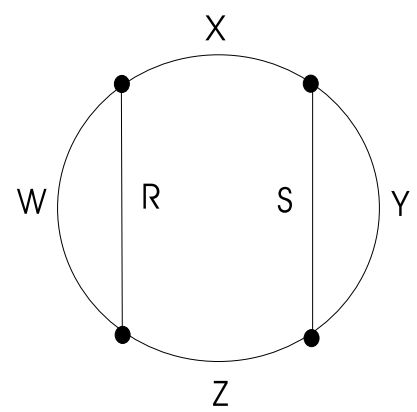

Figure 6: Diagram for the proof of Theorem 8.

It remains to consider the case where $R$ is the only path in $G^{\prime}$ joining two vertices in $C$ and having no internal vertex in $C$. The addition of an ear $F$ joining two vertices of $U \in\{P, Q, R\}$ yields a uniquely determined circuit $D \subseteq F \cup U$. If $U=P$ then $D$ and $R \cup Q$ have the same parity by (4). Moreover $G$ is of type $\mathcal{G}_{1}$ if that parity is even and of type $\mathcal{G}_{2}$ otherwise. We may therefore assume by symmetry that $U=R$. If $D$ is even then $G$ even-contains $2 \mathrm{~A}$ or $2 \mathrm{~B}$ since $C$ is odd. Otherwise $G$ is of type $\mathcal{G}_{2}$.

Concerning statement (3), if $E(G)$ is an even circuit or $G$ is isomorphic to $K_{2}$, then $G$ is alternating-realizable. Suppose on the other hand that $G$ is 1-extendable but that $G$ is not isomorphic to $K_{2}$ and $E(G)$ is not an even circuit. Then some alternating circuit $A$ is a proper subset of $E(G)$. Let $f$ and $g$ be 1-factors of $G$ such that $A=f+g$. Since $A \neq E(G)$ there is an edge $e$ of $E(G)-A$ incident on a vertex of $A$, and since $G$ is 1-extendable $e$ must belong to a 1-factor $h \notin\{f, g\}$. Hence $f+h$ is the union of a set $\mathcal{S}$ of alternating circuits. The sum of the circuits in $\mathcal{S} \cup\{A\}$ is $(f+h)+(f+g)=h+g$, which is the union of another set $\mathcal{T}$ of alternating circuits. The sum of the circuits in $\mathcal{S} \cup \mathcal{T} \cup\{A\}$ being empty, we deduce from Lemma 4 that $G$ is not alternating-realizable.

There is a dual version of Theorem 8. Concerning terminology, if $G$ is a plane graph (a particular planar embedding of a planar graph), then $G^{*}$ denotes its dual graph. Note that, for any connected graph $G$ embedded in the plane, $G^{* *}=G$. If $H$ is a subgraph of the embedded graph, then $H^{*}$ is the subgraph of $G^{*}$ consisting of the dual edges of $H$.

A bond of $G$ is the minimal nonempty edge cut. To each bond of a 2-connected graph $G$ assign a parity - even or odd. If $\mathcal{B}$ denotes the set of all bonds, then this assignment can be considered a function

$$
f: \mathcal{B} \rightarrow \mathbb{Z}_{2}
$$


where 0 is even and 1 is odd. Call $f$ dual realizable if there exists a set $E$ of edges of $G$ such that

$$
|B \cap E| \equiv f(B) \quad(\bmod 2)
$$

for every bond $B \in \mathcal{B}$. Call $G$ dual realizable if every function $f: \mathcal{B} \rightarrow \mathbb{Z}_{2}$ is realizable.

The set $\mathcal{B}$ generates the cocycle space of $G$. Likewise the set $\mathcal{B}_{0}$ of even cardinality bonds generates the even cocycle space. Call $G$ dual even-realizable if every function $f: \mathcal{B}_{0} \rightarrow \mathbb{Z}_{2}$ is dual realizable.

A rope of a (multi)graph $G$ is a subgraph induced by two adjacent vertices. Let $N$ be a rope of $G$ and let $H$ be the graph obtained by contracting the edges of $N$. Then $G$ is said to be obtained from $H$ by a rope addition. A rope decomposition of $G$ is a sequence $G_{0}, G_{1}, \ldots, G_{n}$ of contractions of $G$ such that $G_{0}=K_{1}, G_{n}=G$ and $G_{i}$ is obtained from $G_{i-1}$ by a rope addition for each $i>0$. Our proof of Theorem 15 below is based on the following analog of the well-known Ear Decomposition Theorem.

Lemma 9 Any 2-connected graph has a rope decomposition.

Proof: It is sufficient to show that any 2-connected graph $G$ has a rope $r$ such that the graph $G / r$ obtained by contracting $r$ to a vertex $v_{r}$ remains 2-connected (or has fewer than 3 vertices). Assume not. Then for any rope $r$ of $G$, the graph $G / r$ has at least two blocks. Choose $r$ and a block $B_{r}$ of $G / r$ to minimise the number of ropes of $B_{r}$. Let $s$ be a rope of $B_{r}$. If every rope in $B_{r}$ is incident with vertex $v_{r}$, then $G / s$ is 2-connected, a contradiction. Otherwise assume $s$ is not incident with $v_{r}$. In this case $G / s$ has a block with fewer ropes than $B_{r}$, contradicting the choice of $r$ and $B_{r}$.

A dual subdivision of a graph $G$ is obtained from $G$ by adding any number of multiple edges to existing edges. An even dual subdivision of $G$ is obtained from $G$ by adding an even number of multiple edges to each of any number of existing edges. Two graphs are said to be (even) dual equivalent if they have a common (even) dual subdivision.

Define three families of graphs $\mathcal{G}_{0}^{*}, \mathcal{G}_{1}^{*}, \mathcal{G}_{2}^{*}$ as follows. The family $\mathcal{G}_{0}^{*}$ consists of two vertices joined by any number of edges. The family $\mathcal{G}_{1}^{*}$ consists of all graphs obtained from a graph in $\mathcal{G}_{0}^{*}$ with an odd number of edges by the following procedure. Replace any number of single edges by a path of length 2 , and any number of pairs of edges by copies of the first graph in Figure 7. An example of a graph in $\mathcal{G}_{1}^{*}$ also appears in Figure 7. The family $\mathcal{G}_{2}^{*}$ is represented by the graphs in Figure 8, where the thick line represents a multiple edge (any number of edges including zero). A graph even dual equivalent to a graph from $\mathcal{G}_{0}^{*}, \mathcal{G}_{1}^{*}$ or $\mathcal{G}_{2}^{*}$ will be referred to as a graph of type $\mathcal{G}_{0}^{*}, \mathcal{G}_{1}^{*}$ or $\mathcal{G}_{2}^{*}$, respectively. The following lemmas correspond to those preceding Theorem 8 , and the proofs are similar in most cases.

Lemma 10 A graph is dual (even) realizable if and only if there exists no linearly dependent set of bonds in its (even) cocycle space. 

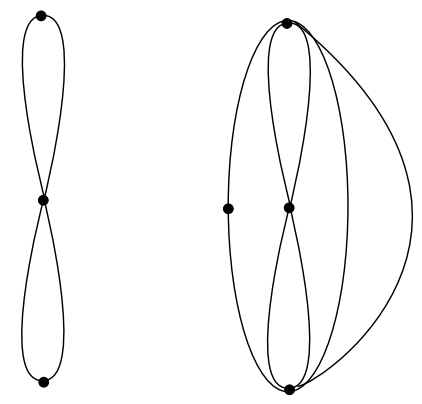

Figure 7: A graph in $\mathcal{G}_{1}^{*}$.
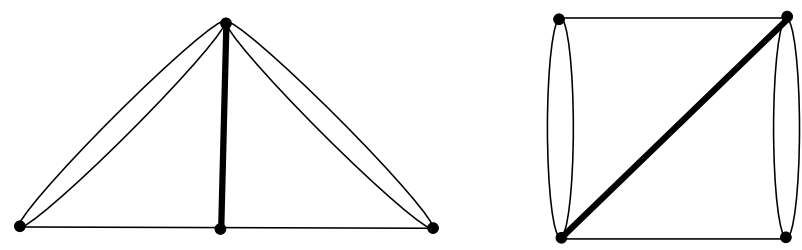

Figure 8: The graphs in $\mathcal{G}_{2}^{*}$.

Lemma 11 Let $G$ be a connected plane graph. Then $G$ is (even) realizable if and only if $G^{*}$ is dual (even) realizable.

Proof: Assume that $G$ is not (even) realizable. Then there exists a dependent set $C$ of (even) circuits in $G$. The set $B$ consisting of the duals of these circuits is a dependent set of (even) bonds in $G^{*}$. The converse is proved similarly.

Lemma 12 The dual of a graph of type $\mathcal{G}_{0}, \mathcal{G}_{1}$ or $\mathcal{G}_{2}$ embedded in the plane is of type $\mathcal{G}_{0}^{*}, \mathcal{G}_{1}^{*}$ or $\mathcal{G}_{2}^{*}$.

Lemma 13 If $G^{\prime}$ is a contraction of $G$, and $G$ is dual (even) realizable, then $G^{\prime}$ is also dual (even) realizable.

Proof: This result follows from Lemma 10 and the fact that each bond of $G^{\prime}$ is a bond of G.

Lemma 14 If $G$ is obtained from a graph of type $\mathcal{G}_{0}^{*}, \mathcal{G}_{1}^{*}$ or $\mathcal{G}_{2}^{*}$ by a rope addition, then $G$ is planar or $G$ is not dual even-realizable.

Proof: If $G$ is obtained from a graph of type $\mathcal{G}_{0}^{*}$ or $\mathcal{G}_{2}^{*}$ by a rope addition, then $G$ is clearly planar.

Let $H$ be a graph of type $\mathcal{G}_{1}^{*}$. Let $G$ be a non-planar graph obtained from $H$ by the addition of a rope $r$. The rope $r$ in $G$, after contraction, corresponds to a vertex $x$ in $H$. Let $u, v$ be the vertices of rope $r$ in $G$. Now $G$ must have the following structure. The set of vertices is $\left\{u, v, y, 1,2, \ldots, n, u_{1}, u_{2}, \ldots, u_{j}, v_{1}, v_{2}, \ldots, v_{k}\right\}, j, k \geq 0$, where 
1. vertices $u$ and $v$ are joined by the rope $r$;

2. $u$ and $v$ are each adjacent to each vertex $1,2, \ldots, n$;

3. $u$ is adjacent to each vertex $u_{1}, u_{2}, \ldots, u_{j}$ (by a single or double edge) and $v$ is adjacent to each vertex $v_{1}, v_{2}, \ldots, v_{k}$ (by a single or double edge);

4. $y$ is adjacent to each vertex $1,2, \ldots, n$ by a double edge;

5. $y$ is adjacent to $u$ by some number (possibly 0) of edges and to $v$ by some number (possibly 0) of edges;

6. each $u_{i}$ is adjacent to $y$ by as many edges as to $u$, and each $v_{i}$ is adjacent to $y$ by as many edges as to $v$;

7. there are no other edges; and

8. $n \geq 3$ since $G$ is non-planar.

Lemma 10 will be used to show that $G$ is not dual even-realizable. Hence it is sufficient to find a dependent set of even bonds in $G$. By the definition of $\mathcal{G}_{1}^{*}$, exactly one of the two vertices $u, v$ has even degree, say vertex $u$. In general, if $W$ is a set of vertices then $\partial W$ denotes the bond such that one component of $G-\partial W$ is the graph induced by $W$. (This assumes that the graphs induced by $W$ and by $V(G)-W$ are connected.) Note that $\partial W=\sum_{w \in W} \partial\{w\}$, where the addition is symmetric difference. In our situation, let $B_{i}=\partial\{u, i\}, i \in\{1,2,3\}$, and $B_{0}=\partial\{u, 1,2,3\}$. Then $B_{0}, B_{1}, B_{2}, B_{3}$ are all even bonds since vertices $u, 1,2,3$ have even degree. Also $B_{0}+B_{1}+B_{2}+B_{3}=\partial\{u)+\partial\{1\}+\partial\{2\}+$ $\partial\{3\}+\partial\{u\}+\partial\{1\}+\partial\{u\}+\partial\{2\}+\partial\{u\}+\partial\{3\}=\emptyset$. Therefore $\left\{B_{0}, B_{1}, B_{2}, B_{3}\right\}$ is a dependent set of bonds.

Theorem 15 Let $G$ be a 2-connected graph.

1. $G$ is dual realizable if and only if $G$ is of type $\mathcal{G}_{0}^{*}$.

2. $G$ is dual even-realizable if and only if $G$ is of type $\mathcal{G}_{0}^{*}, \mathcal{G}_{1}^{*}$ or $\mathcal{G}_{2}^{*}$.

Proof: Concerning statement (1), if $G$ is of type $\mathcal{G}_{0}^{*}$, then $G$ is obviously dual realizable. In the other direction, if $G$ is 2-connected but not of type $\mathcal{G}_{0}^{*}$, then $G$ can be contracted to a circuit of length at least 3 (with possibly multiple edges). But such a circuit is not dual realizable by Lemma 10. By Lemma 13, $G$ is also not dual realizable.

Concerning statement (2), if $G$ is a graph of type $\mathcal{G}_{0}^{*}, \mathcal{G}_{1}^{*}$ or $\mathcal{G}_{2}^{*}$, then it follows from Lemma 10 that $G$ is dual even-realizable. In the other direction, assume that $G$ is dual even-realizable. We must show that $G$ is of type $\mathcal{G}_{0}^{*}, \mathcal{G}_{1}^{*}$ or $\mathcal{G}_{2}^{*}$.

Since $G$ is 2-connected it has a rope decomposition by Lemma 9 . It will now be shown, by induction on the number of ropes in the decomposition of $G$, that $G$ must be of type 
$\mathcal{G}_{0}^{*}, \mathcal{G}_{1}^{*}$ or $\mathcal{G}_{2}^{*}$. If there is just one rope in the decomposition, then $G$ is in $\mathcal{G}_{0}^{*}$. Let $G^{\prime}$ be the second to last stage in the rope decomposition. The addition of one more rope to $G^{\prime}$ will give $G$. Since $G^{\prime}$ is a contraction of $G$, it is dual even-realizable by Lemma 13 . By the induction hypothesis $G^{\prime}$ is of type $\mathcal{G}_{0}^{*}, \mathcal{G}_{1}^{*}$ or $\mathcal{G}_{2}^{*}$. By Lemma 14 the graph $G$ is planar or not dual even-realizable. The latter is not possible, so $G$ is planar. Consider any embedding of $G$ in the plane. Since $G$ is dual even-realizable, $G^{*}$ is even-realizable by Lemma 11. Therefore $G^{*}$ is of type $\mathcal{G}_{0}, \mathcal{G}_{1}$ or $\mathcal{G}_{2}$ by Theorem 8 . By Lemma 12 the graph $G=G^{* *}$ is of type $\mathcal{G}_{0}^{*}, \mathcal{G}_{1}^{*}$ or $\mathcal{G}_{2}^{*}$.

\section{References}

[1] Fischer, I., Little, C.H.C., Even circuits of prescribed clockwise parity, Electronic J. Combin. 10(1) (2003), R45.

[2] Fischer, I., Little, C.H.C., A characterisation of Pfaffian near bipartite graphs, J. Combinatorial Theory B82 (2001) 175-222.

[3] Fischer, I., Little, C.H.C., Rendl, F., Towards a characterisation of Pfaffian near bipartite graphs, Disc. Math. 244 (2002) 279-297.

[4] Hartmann, S., Little, C.H.C., Even bonds of prescribed clockwise parity, preprint.

[5] Kasteleyn, P.W., Graph theory and crystal physics, in F. Harary, ed., Graph Theory and Theoretical Physics, Academic Press, London, 1967, 43-110.

[6] Norine, S., Little, C.H.C., Teo, K.L., A new proof of a characterization of Pfaffian bipartite graphs, J. Combinatorial Theory B91 (2004) 123-126.

[7] Robertson, N., Seymour, P.D., and Thomas, R., Permanents, Pfaffian orientations, and even directed graphs Annals of Math. 150 (1999) 929-975.

[8] McCuaig, W.D., Rosenfeld, M, Parity of cycles containing specified edges, Cycles in Graphs, Annals of Disc. Math., Alspach, Godsil, eds. 27 (1985) 119-132. 